\title{
Big is beautiful? - Zum Stellenwert quantifizierender Betrachtungen und kritischer Reflexion territorialer Größe im politischen Denken der griechisch-römischen Antike
}

\author{
Karen Piepenbrink
}

\section{Einleitung}

Im politischen Denken der griechisch-römischen Antike sind quantifizierende Betrachtungen weitaus weniger stark vertreten als qualifizierende. Wenn sie vorkommen, dann gewöhnlich in der Weise, dass die Relation zwischen Größerem und Kleinerem in den Blick genommen wird, etwa zwischen Gemeinwesen unterschiedlicher Größe bzw. zwischen verschieden großen politisch aktiven Gruppierungen innerhalb eines Gemeinwesens oder aber dergestalt, dass Größe explizit goutiert wird, beispielsweise im Hinblick auf das Ausmaß einer bestimmten Herrschaft. ${ }^{1}$ Eine dezidiert kritische Auseinandersetzung mit Größe oder gar eine positive Konnotation von Kleinem begegnen dagegen weitaus seltener. ${ }^{2}$ Das mag verwundern angesichts der Tatsache, dass dieser Gegenstand in der Rezeption antiker Politik seit der Frühen Neuzeit bis hin zur Auseinandersetzung mit antiken Herrschaftsformen in der heutigen Fachwissenschaft keinesfalls marginal ist. Berühmt ist etwa die im Kontext der Aufklärung mehrfach formulierte Ansicht, dass die partizipatorische Demokratie einzig in kleinen staatlichen Formationen, namentlich Stadtstaaten praktikabel sei, während sie für Flächenstaaten nicht tauge. ${ }^{3}$ Für letztere seien entweder Monarchien probat oder aber republikanische Verfassungen mit einem Repräsentativsystem. ${ }^{4}$ Als Beispiele für eine partizipative Demokratie werden dabei bevorzugt jene der griechischen Poleis angeführt, die zum einen als klein gekennzeichnet werden und denen zum anderen all die Merkmale zugeschrieben werden, die wir aus der antiken Demokratiekritik kennen. ${ }^{5}$

\footnotetext{
${ }^{1}$ Ersteres begegnet insbesondere in der Historiographie in außenpolitischen Kontexten, der zweite Aspekt vorzugsweise in der politischen Philosophie im Zusammenhang mit inneren Konflikten resp. Fragen der Teilhabe an der Macht. Das dritte Moment findet sich wiederum vorrangig in historiographischen Texten, wird im griechischen Bereich etwa in Bezug auf Athen oder auch das Perserreich formuliert, im römischen schwerpunktmäßig mit Blick auf die römische Herrschaft selbst. Einschlägige Stellen werden im Verlaufe des Beitrags angeführt.

${ }^{2}$ Ein Beispiel hierfür wäre die Lobpreisung militärischer Erfolge, die mit vergleichsweisen kleinen Heeren und begrenzten Ressourcen gegen einen übermächtigen Gegner erzielt wurden. Intention dabei ist, die besondere Tapferkeit der betreffenden Heerführer und ihrer Soldaten zu akzentuieren, auf die solche Erfolge zurückgeführt werden; vgl. etwa Sall. Cat. 53,2-5.

${ }^{3}$ Hierzu mit Belegen aus dem Umkreis der Amerikanischen wie auch der Französischen Revolution W. Nippel, Antike oder moderne Freiheit? Die Begründung der Demokratie in Athen und in der Neuzeit, Frankfurt/M. 2008, 113f.; ders., Die Verabschiedung der Antike durch die Französische Revolution, in: K. Ridder/S. Patzold (Hg.), Die Aktualität der Vormoderne. Epochenentwürfe zwischen Alterität und Kontinuität, Berlin 2013, 161-183, bes. 162f.

${ }^{4}$ Letztere Position vertreten insbesondere James Madison und Alexander Hamilton in den ,Federalist Papers'; zu deren Einstellungen und ihrer Verortung im historischen Kontext M.H. Hansen, The Tradition of the Athenian Democracy A. D. 1750 - 1990, in: G\&R 39 (1992) 14-30, hier 23; J.T. Roberts, Athens on Trial. The Antidemocratic Tradition in Western Thought, Princeton/N.J. 1994, 186; W. Heun, Die Antike in den amerikanischen politischen Debatten in der zweiten Hälfte des 18. Jahrhunderts, in: U. Niggemann/K. Ruffing (Hg.) Antike als Modell für Nordamerika? Konstruktion und Verargumentierung 1763-1809, München 2011, 65-83, hier 80f.

${ }^{5} \mathrm{Im}$ Vordergrund steht dabei die Kritik an der Herrschaft einer besitzlosen und vermeintlich politisch inkompetenten Menge.
} 
Selbstredend haben wir es an der Stelle nicht mit einer positiven Würdigung von Kleinheit zu tun: Das Kleine und die direkte Demokratie werden mit dem Attribut der Alterität belegt und von großräumigen Gemeinwesen mit vermeintlich moderner Staatlichkeit abgegrenzt.

Eine andere Überlegung, die bis heute in der Fachwissenschaft wie auch in populären Betrachtungen verbreitet anzutreffen ist, betrifft die sog. ,Überdehnung ‘ des römischen Staates. Sie begegnet in mehreren Varianten: Die eine bezieht sich auf die späte Republik, konkret auf die negativen Konsequenzen der Expansion im Mittelmeerraum. Sie hebt zumeist auf den Umstand ab, dass die römische res publica eine stadtstaatliche Ordnung darstelle, die imperialen Verhältnissen nicht gewachsen sei. Prominent begegnet diese Auffassung etwa im frühen 18. Jahrhundert bei Charles de Montesquieu, ${ }^{6}$ dann wieder in fachwissenschaftlichen Arbeiten des 19. und beginnenden 20. Jahrhunderts, in denen die Implementierung monarchischer Strukturen gern als unausweichlich gezeichnet wird, um die Größe des Herrschaftsraumes bewältigen zu können, durchaus aber auch noch in der modernen Forschung. ${ }^{7}$ Der Prinzipat erscheint angesichts dessen zunächst als Lösung, zumindest bis ins späte zweite Jahrhundert. Mit Blick auf die nachfolgende Zeit, insbesondere die sog. Krise des dritten Jahrhunderts wie auch die gesamte Spätantike, wird abermals gern auf das Paradigma der ,Überdehnung' rekurriert, heutzutage vor allem in populärwissenschaftlichen Beiträgen. ${ }^{8}$ Aus der Vergangenheit haben wir nichtsdestotrotz illustre Beispiele hierfür, darunter Edward Gibbons Überlegungen zum Verfall des Römischen Reiches. Gibbon arbeitet eine ganze Reihe von Aspekten heraus, die er teils auf die Christianisierung, teils auf die übermäßige Ausdehnung des Reiches zurückführt. ${ }^{9}$ Die allermeisten Kriterien, die er anführt, finden sich bereits in der Antike, namentlich bei spätantiken paganen Autoren - ausgenommen die Größenproblematik. ${ }^{10}$

Dieser Befund ist bezeichnend. Ich möchte dem Phänomen im Folgenden näher nachgehen und dazu das politische Denken der griechisch-römischen Welt im Hinblick auf mögliche Problematisierungen von Größe durchforsten. Mir geht es dabei nicht um eine Materialsammlung mit Vollständigkeitsanspruch, sondern um eine typologische Betrachtung einschlägiger Beispiele und deren Interpretation.

\section{Beispiele aus dem griechischen Raum}

Beginnen wir mit der griechischen Poliswelt: In dieser stoßen wir in der klassischen Zeit auf eine Reihe positiver Belege. Ein markantes Sujet des griechischen politischen Denkens, u. a. der Historiographie und der politischen Philosophie der Zeit, ist die

\footnotetext{
${ }^{6} \mathrm{Ch}$. de Montesquieu, Considérations sur les causes de la grandeur des Romains et leur décadence, Amsterdam 1734, Kap. 9.

${ }^{7}$ Eine Skizze zu diesem Ansatz in der modernen Forschung gibt K. Bringmann, Krise und Ende der römischen Republik (133-42 v. Chr.), Berlin 2003, 38-44; auch E. Baltrusch, Außenpolitik, Bünde und Reichsbildung in der Antike, München 2008, 166.

${ }^{8}$ Siehe beispielsweise P. Heather, The Fall of the Roman Empire. A New History, London 2005, 103110.

${ }^{9}$ E. Gibbon, The History of the Decline and Fall of the Roman Empire, London 1781, Bd. 3, Kap. 38; zu dessen Vorstellungen und ihrer Kontextualisierung K. Christ, Der Untergang des römischen Reiches in antiker und moderner Sicht. Eine Einleitung, in: ders. (Hg.), Der Untergang des römischen Reiches, Darmstadt 1970, 1-31, hier 11f.

${ }^{10}$ Die vermeintlichen Konsequenzen der Christianisierung, die er auflistet und als Verfallsgründe interpretiert, begegnen nachgerade bei Zosimos; dazu mit Belegen A. Demandt, Der Fall Roms. Die Auflösung des römischen Reiches im Urteil der Nachwelt. Erweiterte und aktualisierte Neuauflage München $2014,45-55$.
} 
Konstituierung von Poleis. Es begegnet in Berichten über die Einrichtung von $\dot{\alpha} \pi$ oıkíl, in ätiologischen Reflexionen über die Anfänge von Städten oder auch im Kontext der Frage, wie ein Städtegründer oder Nomothet zu verfahren hat, der eine trefflich verfasste Polis kreieren bzw. ausgestalten möchte. Hier geht es nicht nur um Herrschaftsinstitutionen, sondern auch um die Anlage der Stadt. Ein zentraler Aspekt dabei ist deren Größe, d. h. die Ausmaße des Territoriums, speziell aber die Anzahl der Bewohner. In dem Zusammenhang stoßen wir auf Überlegungen, die auf Sensibilität dafür schließen lassen, dass es ein ,zu groß $\beta^{\star}$ geben kann, und zwar insbesondere im Hinblick auf die Bevölkerungszahlen. Als Ideal erscheint, wenn eine Polis in der Lage ist, ihre Einwohner vollumfänglich selbst zu versorgen und sich damit durch ,Autarkie' auszeichnet. Wir finden den Gedanken vorrangig in der politischen Philosophie, namentlich in Platons Politeia und seinen Nomoi sowie den aristotelischen Politika. ${ }^{11}$ Gleichwohl ist der Terminus im politischen Denken nicht stark verbreitet, im Unterschied etwa zur mit ihm verwandten Kategorie der ,Autonomie'. ${ }^{12}$ Sowohl Platon als auch Aristoteles gehen davon aus, dass einzig eine kleine Polis autark zu sein vermag. Auf der Basis heutigen ökonomischen Denkens mag das verwundern wir würden eher die Relation zwischen Bedarfen und wirtschaftlicher Leistungsfähigkeit in den Blick nehmen als die absolute Kleinheit -; ausgehend vom Erfahrungsraum der beiden Philosophen aber lässt sich ihre Annahme unschwer nachvollziehen: Sie sind sich der Tatsache bewusst, dass die Poleis der eigenen Zeit im Normalfall nicht autark sind. In Übereinstimmung mit der Mehrzahl ihrer Zeitgenossen setzen sie voraus, dass die Poleis im Verlauf der Zeit an Größe zugenommen und zugleich einen sozialen Differenzierungsprozess durchlaufen haben, in dem sich Eliten mit exquisitem Lebensstil und entsprechenden Konsumbedürfnissen herausgebildet haben, die selbst große Poleis nur durch Importe zu befriedigen vermögen. Die autarke Polis begreifen sie demgegenüber als eine Frühform in der Genese des Stadtstaates, für die tatsächlich weniger das Moment der geringen Größe konstitutiv ist als das der Selbstbeschränkung der Bewohner. Sie operieren damit schlussendlich doch eher mit qualitativen als mit quantitativen Kategorien.

Mit der Frage nach der rechten Größe der Polis beschäftigt sich Aristoteles zudem im Kontext seiner Auseinandersetzung mit der bestmöglichen politischen Ordnung. Sein Augenmerk gilt dabei jeweils der Einzelpolis und deren Stabilisierung angesichts der Bedrohung durch innere Konflikte. Hier interessiert er sich u. a. für die adäquate Zahl an Bürgern. Er formuliert die These, dass es für eine Polis mit großer Bürgerzahl ausnehmend schwierig sei, eine gute Verfassung zu bewahren. ${ }^{13}$ Wohlgeordneten Städten attestiert er im Umkehrschluss, gewöhnlich sorgsam darüber zu wachen, dass die Zahl ihrer Bürger nicht unkontrolliert wachse. An der Stelle denkt er zuvorderst an die restriktive Handhabung des Bürgerrechts und konzipiert die Polis als face-to-face-community: Die Bürger müssten miteinander vertraut sein, um Rechtsfälle korrekt entscheiden und Amtsträger kompetent bestellen zu können. Gegenseitiges Kennen verhindere außerdem, dass jemand das Bürgerrecht usurpiere und

\footnotetext{
${ }^{11}$ In der Politeia lässt Platon die Gesprächspartner zunächst eine ,einfache' Stadt entwerfen, um diese dann zu einer ,üppigen` ( $\tau \rho \varphi \tilde{\omega} \sigma \alpha \pi \delta ́ \lambda \iota \varsigma)$ anwachsen zu lassen (Plat. rep. 369 b - 372 c); zu seinem Gedankengang J.T. Scott, Aristotle and the ,City of Sows'. Doing Justice to Plato, in: L.G. Rubin (Hg.), Justice v. Law in Greek Political Thought, Lanham/MD 1997, 41-68, hier 47-53. In den Nomoi reflektiert er die Lebensbedingungen sowie die Herausbildung von Herrschaft nach der großen Flut; siehe bes. Plat. nom. 677 a -682 e. Aristoteles beschäftigt sich mit der Thematik im Rahmen seiner grundsätzlichen Überlegungen zur Genese der politischen Gemeinschaft und ihrem Verhältnis zu den vorstaatlichen Gemeinschaften zu Beginn des ersten Buches der ,Politik‘.

${ }^{12}$ Einen Überblick bietet P. Wilpert, Art. Autarkie, in: RAC 1 (1950) Sp. 1039-1050.

${ }^{13}$ Aristot. pol. 1326 a 25-28.
} 


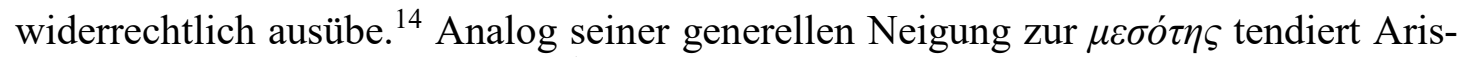
toteles zu einer mittleren Größe. ${ }^{15}$ Er zeigt sich allerdings wenig optimistisch, damit auf Zustimmung zu stoßen, sondern nimmt an, dass die Mehrzahl seiner Zeitgenossen große Poleis favorisierte. ${ }^{16}$

Damit dürfte er richtigliegen; auch im politischen Denken haben wir dahingehende Hinweise, insbesondere in Texten, die sich nicht auf die Einzelpolis kaprizieren, sondern auch die zwischenstaatliche Ebene in die Betrachtungen einbeziehen. Das gilt nachgerade für die Geschichtsschreibung, nicht zuletzt das Werk des Thukydides. Auch der Historiograph geht von einem Prozess zunehmender Machtbildung in der griechischen Welt aus, in dem es großen Poleis wiederholt gelungen ist, hegemoniale Positionen für sich zu reklamieren und kleinere unter ihre Verfügungsgewalt zu bringen. Aus Sicht der kleinen Poleis bewertet er diese Entwicklung ambivalent: Sie büßen an Autonomie ein, gewinnen aber im Gegenzug Schutz gegenüber Angriffen durch dritte. ${ }^{17}$ Bezeichnend ist an der Stelle der Melier-Dialog: Dort wird deutlich, dass die Option, dass eine vergleichsweise kleine politische Einheit für sich Neutralitätsstatus reklamiert, prinzipiell denk- und beschreibbar ist. ${ }^{18}$ Aus der Sicht des Hegemons Athen ist sie freilich nicht tolerierbar. ${ }^{19}$ Die Athener operieren mit dem Freund-Feind-Schema sowie dem Recht des Stärkeren; ein Existenzrecht für kleine Poleis, die Souveränität für sich beanspruchen, sehen sie nicht vor. ${ }^{20}$

Allerdings reflektiert Thukydides durchaus, dass Größe auch mit Problemen einhergehen kann, und zwar nicht nur für kleinere Poleis, die dem Dominanzstreben größerer ausgesetzt sind, sondern ebenso für die größeren selbst. Im Falle Athens bemerkt er mit Blick auf die Sizilische Expedition, dass die Größe der Stadt und das damit verbundene Ausmaß ihrer Herrschaft zu Selbstüberschätzung und Fehlentscheidungen geführt hätten. ${ }^{21} \mathrm{Er}$ nimmt hier jedoch keinen autonomen Prozess an, der zwangsläufig mit Wachstum vergesellschaftet sei, sondern vertritt die Position, dass verantwortungsvolle Politiker nach dem Muster der Perikles derartigen Entwicklungen entgegenzuwirken imstande seien. ${ }^{22}$ Ein Politiker dieser Art wird nach Thukydides gleichwohl nicht für einen Verzicht auf Expansion oder gar die Verkleinerung des Herrschaftsgebietes plädieren, sondern für ein moderates Wachstum, das den jeweiligen Handlungsbedingungen Rechnung trägt.

Ein anderer für unsere Thematik relevanter Gedanke, der bei Thukydides begegnet, aber auch schon bei Herodot vorkommt und in der Folgezeit in verschiedenen literarischen Gattungen immer wieder auftaucht, ist der von der Unsicherheit aller Machtbildungen und sämtlicher äußeren Güter. ${ }^{23}$ Poleis, die lange vom Glück begünstigt scheinen, Macht und Wohlstand zu erringen vermochten, laufen Gefahr, all des-

\footnotetext{
${ }^{14}$ Aristot. pol. 1326 b 11-25. Außerdem gibt er zu bedenken, dass es einem Feldherrn Schwierigkeiten bereiten könne, stimmlich durchzudringen, wenn das Heer allzu stark anwachse; gleiches gelte für den Herold, der für die Ordnung in der Volksversammlung zu sorgen hat.

15 Aristot. pol. 1326 a $35-1326$ b 5 .

${ }^{16}$ Daneben kalkuliert er die - etwa von Platon vertretene - Position ein, dass jemand für Kleinräumigkeit plädiert; zu dem Komplex Aristot. pol. 1326 b 36-39.

${ }^{17}$ Siehe Thuk. 1,2; vgl. ibid. 1,8,3.

18 Thuk. 5,94. 96.98.

19 Thuk. 5,95. 97. 99.

${ }^{20}$ Aus politologischer Sicht ist das insbesondere auf die imperiale Perspektive der Athener zurückzuführen, welcher der Gedanke der Koexistenz souveräner Staaten fremd ist, zumindest bei asymmetrischen Verhältnissen; dazu H. Münkler, Imperien. Die Logik der Weltherrschaft - vom Alten Rom bis zu den Vereinigten Staaten, Berlin 2005, 30f.

${ }^{21}$ Thuk. 2,65,11.

22 Thuk. 2,65, 5. 8f.

${ }^{23}$ Siehe z. B. Hdt. 1,5,4; Thuk. 4,18.
} 
sen wieder verlustig zu gehen. Keine Stadt kann sich einmal erlangter Größe auf Dauer sicher sein. Die Ursachen hierfür werden jedoch nicht in der Größe ausgemacht, sondern mit dem Zufall oder Schicksal in Verbindung gebracht. ${ }^{24}$ Andere Überlegungen zum Verfall bzw. zum Aufstieg und Niedergang von Herrschaften aus dem griechischen Kulturkreis sind stärker durch die Rechtsidee bzw. die Orientierung an kooperativen Werten geprägt. ${ }^{25}$ Auch diese problematisieren nicht die übermäßige Größe von Gemeinwesen, sondern ein Übermaß an Bedürfnissen auf Seiten der Herrschenden. Lösungsansätze sehen sie demzufolge am ehesten in der Selbstbeschränkung der betreffenden Personen.

All das gilt grundsätzlich auch noch für den Hellenismus, in dem die Thematik jedoch mit der Verschiebung der Perspektive auf größere territoriale Einheiten an Bedeutung einbüßt. Die Etablierung letzterer wird von den Zeitgenossen weniger unter dem Aspekt nachhaltiger Sicherung, als vielmehr unter dem Gesichtspunkt der ,Sieghaftigkeit' beleuchtet. ${ }^{26}$ Kritische Reflexionen, die quantitative Phänomene anvisieren, bleiben damit aus. Hinzu kommt die Fokussierung auf Herrscherpersönlichkeiten, die nicht zuletzt zur Konsequenz hat, dass organisatorisch-institutionelle Momente gegenüber ethischen im politischen Denken weiter noch als bislang schon zurücktreten. ${ }^{27}$

\section{Beispiele aus der römischen Welt}

Im politischen Denken der römischen Welt wird die Größe von Herrschaftsbereichen noch deutlich weniger problematisiert als im griechischen Kulturraum der klassischen Zeit. Die Vorstellung von einem historischen Prozess, der durch Machtbildung und zunehmende Machtkonzentration gekennzeichnet ist, wird von römischen Autoren aufgegriffen und auf die eigene res publica transferiert - so beispielsweise von Sallust, Cicero oder Livius. ${ }^{28}$ Sie verknüpfen diese mit spezifisch römischen Aspekten, so der Qualität der mores oder dem Gedanken der göttlichen providentia. ${ }^{29}$ Das Moment des Zufalls im Sinne der griechischen $\tau \dot{\chi} \chi \eta$ tritt entsprechend in den Hintergrund. ${ }^{30}$ Unsere Autoren gehen vielmehr von einer Disposition der Römer zur Errichtung einer großräumigen Herrschaft aus, die sie aus deren virtutes, aber auch der Be-

\footnotetext{
${ }^{24}$ So etwa Thuk. 4,18.

${ }^{25}$ Den Anfang greifen wir im ,Weltzeitaltermythos' Hesiods (Hes. erg. 106-200), der wohl orientalische Vorbilder verarbeitet und stark durch den Rechtsgedanken geprägt ist. Von organisierter Herrschaft geht er allerdings noch nicht aus.

${ }^{26}$ Dies korreliert mit dem Charakter des charismatischen Königtums; dazu H.-J. Gehrke, Der siegreiche König. Überlegungen zur hellenistischen Monarchie, in: AKG 64 (1982) 247-277. Ein spezifisches Sujet in dem Zusammenhang bildet der Alexanderzug und dessen räumliche Dimensionierung, dazu u. a. H. Sonnabend, Die Grenzen der Welt. Geographische Vorstellungen der Antike, Darmstadt 2007, $115-123$.

${ }^{27}$ Einschlägig sind diesbezüglich insbesondere die Schriften $\pi \varepsilon \rho \grave{\imath} \beta \alpha \sigma l \lambda \varepsilon i \alpha \varsigma ;$ dazu M. Haake, Warum und zu welchem Ende schreibt man peri basileias? Überlegungen zum historischen Kontext einer literarischen Gattung im Hellenismus, in: K. Piepenbrink (Hg.), Philosophie und Lebenswelt in der Antike, Darmstadt 2003, 83-138.

${ }^{28}$ Hierzu mit Quellenbelegen P.A. Brunt, Laus Imperii, in: P.D.A. Garnsey/C.R. Whittaker (Hg.), Imperialism in the Ancient World, Cambridge 1978, 159-191, hier 164-168.

${ }^{29}$ So bereits mit Blick auf die römischen Anfänge Liv. 1,9,3f.

${ }^{30} \mathrm{Zu}$ Unterschieden im römischen fortuna-Verständnis gegenüber griechischen $\tau \dot{\chi} \chi \eta$-Konzeptionen in dem Zusammenhang I. Kajanto, Fortuna, in: ANRW II 17,1 (1981) 502-558, hier 525-532. Der Gedanke des ,Zufalls' im Hinblick auf die römische Machtbildung tritt schon bei Polybios in den Hintergrund; dazu F.K. Maier, „Überall mit dem Unerwarteten rechnen“. Die Kontingenz historischer Prozesse bei Polybios, München 2012, 245-248.
} 
günstigung seitens der Götter herleiten. ${ }^{31}$ Die Vorstellung, eine dezidierte Herrschaftskonzeption entwickeln zu können, die auch Überlegungen zu einer adäquaten Größe des Raumes einschließt, ist ihnen fremd.

Handeln sie über die Begründung städtischer Siedlungen, beschäftigen sie sich in geringerem Umfang mit den Ausmaßen des Territoriums als griechische Denker. Sprechen sie über die Anlage der Stadt, gilt ihr Interesse allem voran deren geographischer Lage, speziell der adäquaten Entfernung zum Meer. ${ }^{32}$ Eine Begrenzung hinsichtlich der Größe bzw. der Bevölkerungszahl ist gewöhnlich nicht intendiert. Dies korreliert mit ihrem Verständnis des Bürgerrechts, das im Unterschied zum griechischen nicht polisgebunden ist und weitaus generöser gehandhabt wird. Signifikant sind in diesem Zusammenhang die Ausführungen des Livius zur Gründung der Stadt Rom: Er berichtet, dass die Mauern mehr in der Hoffnung auf eine künftige Bevölkerung als im Hinblick auf die Menschen errichtet wurden, die damals in der Stadt lebten. ${ }^{33}$ Romulus schreibt er gar gezielte Maßnahmen zur Peuplierung zu, darunter die Einrichtung des sog. asylum. ${ }^{34}$

Die Probleme im Zusammenhang mit der Expansion in der späten Republik werden von zeitgenössischen lateinischen Autoren gewöhnlich nicht ursächlich auf die Größe des Herrschaftsbereiches zurückgeführt und damit nicht auf Schwierigkeiten bei der administrativen Ausgestaltung der Provinzen oder der Kommunikation zwischen provinzialen Statthaltern und stadtrömischen Institutionen. Sallust etwa macht die Ursache bekanntlich in der fortschreitenden sittlichen Devianz innerhalb der Führungsschicht aus, die er vor allem mit der Zerstörung Karthagos und dem Verlust des metus hostilis in Konnex bringt. ${ }^{35}$ Die immensen Ausmaße der Herrschaft erscheinen ihm in Anbetracht dessen gar von Vorteil. So führt er in De Coniuratione Catilinae aus, dass die res publica gerade aufgrund ihrer Größe (magnitudine) die Fehler ihrer Heerführer und Magistrate bislang zu ertragen vermocht habe. ${ }^{36}$ Den Verlust der Eintracht und die Abkehr vom mos maiorum assoziiert er zwar mit dem, Wüten' der fortuna, betrachtet den Verfall aber nicht als unabwendbar. ${ }^{37}$ Es scheint ihm möglich gegenzusteuern, sofern die Oberschicht sich auf ihre früheren Verhaltensmuster besinnt. ${ }^{38}$

Auch Livius bringt die vermeintlichen Dekadenzphänomene mit dem Verlust des Konsenses innerhalb des populus Romanus in Verbindung. ${ }^{39}$ Er generalisiert dabei

\footnotetext{
${ }^{31}$ Der Gedanke begegnet bereits bei Polybios und wird dann von römischen Autoren fortgeschrieben; eine maßgebliche Rolle für die Übernahme des polybianischen Gedenkengutes kommt dabei Cicero zu; dazu mit Stellenangaben Kajanto, Fortuna, 534-536.

${ }^{32}$ Siehe z. B. Cic. rep. 2,5-7; Vitruv empfiehlt für eine Stadtanlage die Wahl eines besonders gesunden Platzes (electio loci saluberrimi) und beleuchtet unter dem Aspekt wiederum auch die Lage zum Meer (Vitruv 1,4,1).

${ }^{33}$ Liv. $1,8,4$

${ }^{34}$ Liv. 1,8,4f.; zu dem Ansatz und seiner Bedeutung für die römische Identitätsbildung E. Dench, Romulus' Asylum. Roman Identities from the Age of Alexander to the Age of Hadrian, Oxford 2005, 362-368.

35 Zur Verknüpfung des Motivs des Sittenverfalls mit dem Gedanken der Integrität des Reiches B. Dunsch, Variationen des metus-hostilis-Gedankens bei Sallust (Cat. 10; Iug. 41; Hist. 1, fr. 11 und 12 M.), in: Grazer Beiträge 25 (2006) 201-217, hier 216.

${ }^{36}$ Sall. Cat. 53,5.

${ }^{37} \mathrm{Zu}$ seinem fortuna-Verständnis an der Stelle B. Biesinger, Römische Dekadenzdiskurse. Untersuchungen zur römischen Geschichtsschreibung und ihren Kontexten (2. Jahrhundert v. Chr. bis 2. Jahrhundert n. Chr.), Stuttgart 2016, 110f.

${ }^{38} \mathrm{Zu}$ seinen Überlegungen zur Sicherung auch weit ausgedehnter imperialer Herrschaft K. Heldmann, Sallust über die römische Weltherrschaft. Ein Geschichtsmodell im ,Catilina' und seine Tradition in der hellenistischen Historiographie, Stuttgart 1993, bes. 54-69.

${ }^{39}$ Liv. praef. 11.
} 
noch stärker als Sallust und macht die Entwicklung weniger an konkreten Ereignissen wie der Zerstörung Karthagos fest. ${ }^{40}$ Wenn er die Ausdehnung des Reiches problematisiert, so geschieht das auch bei ihm unter Rekurs auf das Paradigma des Verfalls der Sitten. ${ }^{41}$ Gegenstand der Betrachtung ist damit nicht der politische Handlungsrahmen oder dessen administrative Gestaltung, sondern die ethische Orientierung der verantwortlichen Akteure. ${ }^{42}$

Grundlegende Alternativen zur bisherigen Politik scheinen unseren Autoren nicht vorstellbar, was zum einen mit der generellen Fokussierung des mos maiorum und der Vergangenheit zu tun hat, zum anderen aber auch - darauf hat vor allem Christian Meier hingewiesen - mit der Tatsache, dass ein Großteil der römischen Bürger von der Entwicklung real profitiert hat. ${ }^{43}$ Die Expansion an sich wird gewöhnlich positiv gewürdigt, selbst in der Endphase der Republik in den Reaktionen auf Pompeius und sogar Caesar. Cicero etwa rühmt letzteren, mit der Überquerung des Kanals nach Britannien an die Grenzen der bewohnten Welt vorgestoßen zu sein. ${ }^{44}$ Caesar agiert dabei dem zeitgenössischen Verständnis nach grundsätzlich im Rahmen der römischen Tradition, d. h. im Kontext des Wettbewerbs innerhalb der Nobilität. ${ }^{45}$

Der Befund zur Kaiserzeit ist komplexer und wird in der Forschung entsprechend kontroverser diskutiert. Das betrifft die konkrete Politik einschließlich der Selbstrepräsentation der principes, das politische Denken der Zeit wie auch die Relation zwischen den beiden Größen. Konsens besteht dahingehend, dass die reale Ex-

\footnotetext{
${ }^{40}$ Siehe bes. Liv. praef. 9. Die diesbezüglichen Unterschiede in den Ausführungen Sallusts und Livius“ sind in der Forschung u. a. mit deren Orientierung an verschiedenen Quellen (an Poseidonios resp. Polybios) in Zusammenhang gebracht worden; zur Debatte darüber D.C. Earl, The Political Thought of Sallust, Cambridge 1961, 42f.; T.C. Luce, Livy. The Composition of his History, Princeton 1977, 4159; U. Hackl, Poseidonios und das Jahr 146 v. Chr. als Epochendatum in der antiken Historiographie, in: Gymnasium 87 (1980) 151-166, bes. 154f.; Dunsch, Variationen des metus-hostilis-Gedankens bei Sallust, 204; Biesinger, Römische Dekadenzdiskurse, 175-178. 232f.

${ }^{41}$ Dies gilt grundsätzlich auch für die Anmerkung des Valerius Maximus, der im Kontext seiner Beschäftigung mit der moderatio berichtet, dass der jüngere Scipio Africanus anlässlich des Sühneopfers am Ende seiner Zensur, als der Schreiber die offizielle Gebetsformel zur Bitte um Verbesserung der Lage des Volkes und Mehrung von dessen Macht vortrug, bemerkt haben soll, die Lage sei ausreichend gut und die Macht groß genug (Val. Max. 4,1,10); zu der Szene R. Pfeilschifter, Eroberung und Debatte. Diskutierten die Römer über ihre Expansion?, in: Ch. Lundgreen (Hg.), Staatlichkeit in Rom? Diskurse und Praxis (in) der römischen Republik, Stuttgart 2014, 141-162, hier 141f. An der Stelle kommen überdies typische Vorstellungen der Kaiserzeit zum Tragen (dazu unten).

${ }^{42} \mathrm{Zu}$ dem Komplex A.W. Lintott, Imperial Expansion and Moral Decline, in: Historia 21 (1972) 626638; K. Bringmann, Weltherrschaft und innere Krise Roms im Spiegel der Geschichtsschreibung des zweiten und ersten Jahrhunderts v. Chr., in: A\&A 23 (1977) 28-49.

${ }^{43}$ C. Meier, Res publica amissa. Eine Studie zu Verfassung und Geschichte der späten römischen Republik, Frankfurt/M. ${ }^{3} 1997$ ( $\left.{ }^{1} 1966\right)$, bes. 201-205; in dem Sinne auch J. v. Ungern-Sternberg, Weltreich und Krise: Äußere Bedingungen für den Niedergang der römischen Republik, in: MusHelv 39 (1982) 254-271, bes. 254f.; W. Nippel, Macht, Machtkontrolle und Machtentgrenzung. Zu einigen antiken Konzeptionen und ihrer Rezeption in der frühen Neuzeit, in: J. Gebhardt/H. Münkler (Hg.), Bürgerschaft und Herrschaft. Zum Verhältnis von Macht und Demokratie im antiken und neuzeitlichen politischen Denken, Baden-Baden 1993, 58-78, bes. 61; B. Linke, Die unfassbare Republik. Idealvorstellungen der Römer, in: U. Niggemann/K. Ruffing (Hg.), Antike als Modell in Nordamerika? Konstruktion und Verargumentierung 1763-1809, München 2011, 37-64, hier 37f.

${ }^{44}$ Vgl. Cic. Balb. 64; hierzu und zu ähnlichen Einschätzungen bei Cassius Dio und Plutarch R. Schulz, Roms Eroberung des Mittelmeeres und der Vorstoß in den Atlantik. Reaktionen, Rechtfertigungen und Rückwirkungen auf die Ideologie, Geographie, Ethnographie und Anthropologie der späten Republik und frühen Kaiserzeit, in: ders. (Hg.), Aufbruch in neue Welten und neue Zeiten. Die großen maritimen Expansionsbewegungen der Antike und Frühen Neuzeit im Vergleich, München 2006, 29-50.

${ }^{45}$ Dazu u. a. Schulz, Roms Eroberung des Mittelmeeres und der Vorstoß in den Atlantik, 37.
}

http://www.fera-journal.eu 
pansion seit der Endphase der Regentschaft des Augustus rückläufig ist. ${ }^{46}$ Eine $\mathrm{Ab}$ kehr vom Anspruch auf Beherrschung des orbis terrarum, wie ihn nachgerade Augustus proklamiert hat, ${ }^{47}$ aber bedeutet das nach Einschätzung der Mehrzahl der Forscher nicht. ${ }^{48}$ Selbst wenn man - wie etwa in der Politikwissenschaft gern praktiziert - von einem Übergang von der ,Expansions'- zu einer ,Konsolidierungsphase“ im Rahmen der imperialen Politik sprechen möchte, ${ }^{49}$ bleibt das Motiv der propagatio imperii virulent. Das hat nicht zuletzt damit zu tun, dass der princeps sich $u$. a. durch Sieghaftigkeit auszuzeichnen hat, ${ }^{50}$ um den Erwartungen der Akzeptanzgruppen zu entsprechen. ${ }^{51}$ Hinzu kommt der Gedanke des Sendungsbewusstseins, der nun, wo das Imperium als Ganzes zum Handlungsfeld eines einzelnen Regenten wird, sogar noch nachdrücklicher artikuliert wird als in der späten Republik. ${ }^{52}$ Von einer konzeptionellen imperialen Strategie, welche die Ausdehnung des Herrschaftsgebietes in die Überlegungen einbezieht, kann jedoch auch jetzt noch keine Rede sein. ${ }^{53}$ Dies ist $u$. a. darauf zurückzuführen, dass ein Verständnis von Außenpolitik, die sich von innerer Politik abgrenzen ließe, nicht existiert. Die Erwartungen an den Kaiser umschließen sämtliche Handlungsfelder und lassen eine solche Dichotomisierung nicht zu. ${ }^{54}$

Im Hinblick auf den Umgang mit äußeren Feinden prädominiert in der Republik wie in der Kaiserzeit die Vorstellung, dass solche eine Bedrohung darstellen. Dies wird besonders dadurch betont, dass man externe Feinde gern als ,Barbaren“

${ }^{46} \mathrm{Zu}$ den Details T. Cornell, The End of Roman Imperial Expansion, in: J. Rich/G. Shipley (Hg.), War and Society in the Roman World, London - New York 1993, 139-170, hier 139f.; W.V. Harris, Roman Power, A Thousand Years of Empire, Cambridge 2016, 114.

${ }^{47}$ Besonders explizit praktiziert er dies in den Res Gestae (Aug. Mon. Anc. 26-33); zu seinem Anspruch u. a. A. Lintott, What was the ,Imperium Romanum“? in: G\&R 28 (1981) 53-67, hier 63; R. Alston, Augustan Imperialism, in: D. Hoyos (Hg.), A Companion to Roman Imperialism, Leiden Boston 2013, 197-211, hier 203-207.

${ }^{48}$ Zur Kontroverse hierüber P.A. Brunt, Augustan Imperialism, in: JRS 53 (1963) 170-176, passim; ders., Roman Imperial Illusions, in: ders., Roman Imperial Themes, Oxford 1990, 433-480, hier 468f.

${ }^{49}$ Vgl. M. Doyle, Empires, Ithaca/NY - London 1984, 93-97; Münkler, Imperien, 80.

50 Zur entsprechenden Repräsentation der Kaiser speziell mit Blick auf den lateinischen Westen C.F. Noreña, Imperial Ideals in the Roman West. Representation, Circulation, Power, Cambridge 2011, 146-165.

${ }^{51}$ Die Felder auf denen er sich zu bewähren hat, unterscheiden sich nicht grundlegend von denen der Angehörigen der Nobilität in der ausgehenden Republik; zu den diesbezüglichen Kontinuitäten S. Mattern, Rome and the Enemy. Imperial Strategy in the Principate, Berkeley - Los Angeles - London 1999, 194-202; zur Bestimmung von Akzeptanzgruppen und zur Beschreibung des Prinzipats als ,Akzeptanzsystem` E. Flaig, Den Kaiser herausfordern. Die Usurpation im Römischen Reich, Frankfurt/M. - New York 1992, bes. 174-207.

${ }^{52}$ Zum Anspruch auf, Weltherrschaft' und dem zugehörigen Forschungsstand Baltrusch, Außenpolitik, Bünde und Reichsbildungen in der Antike, 171.

${ }^{53}$ Zur Debatte über die Existenz einer imperialen Strategie E. Luttwak, The Grand Strategy of the Roman Empire. From the First Century A. D. to the Third, Baltimore 1976, bes. 74f.; F. Millar, Emperors, Frontiers and Foreign Relations, 31 B. C. to A. D. 378, in: Britannia 13 (1982) 1-23, hier 21f.; A. Ferrill, The Grand Strategy of the Roman Empire, in: P. Kennedy (Hg.), Grand Strategies in War and Peace, New Haven - London 1991, 71-85; mit Konzentration auf Augustus E.S. Gruen, The Imperial Policy of Augustus, in: K.A. Raaflaub/M. Toher (Hg.), Between Republic and Empire. Interpretations of Augustus and His Principate, Berkeley - Los Angeles - Oxford 1990, 395-416, hier 395f.; ders. The Expansion of the Empire under Augustus, in: A.K. Bowman/E. Champlin/A. Lintott (Hg.), The Cambridge Ancient History. Bd. 10: The Augustan Empire, 43 B. C. - A. D. 69, Cambridge ${ }^{2} 2008\left({ }^{1} 1996\right)$, 147-197, hier 188f.

${ }^{54}$ Vgl. C. Wendt, Sine fine. Die Entwicklung der römischen Außenpolitik von der späten Republik bis in den frühen Prinzipat, Berlin 2008, bes. 134-140. 180. 
konzeptualisiert und damit als grundsätzlich anders markiert. ${ }^{55}$ Unabhängig davon, wie man die römische Politik aus heutiger Sicht beschreibt - ob man der These vom ,defensiven Imperialismus ' folgt oder die aggressiven Momente bzw. universalistischen Tendenzen in den Vordergrund rückt,$-{ }^{56}$ ist zu konstatieren, dass die Römer in hohem Maße auf Sekurität bedacht waren und Risikopotentiale auszuschalten oder zu marginalisieren suchten. ${ }^{57}$ Der Fokus ist damit auf den realen Gegner jenseits der Grenze gerichtet, nicht auf das Phänomen der Grenze an sich oder mögliche Optionen für künftige Grenzziehungen. Auch wenn die Grenze in der praktischen Politik im Kontext des Ausbaus der Befestigungsanlagen zunehmend ins Visier gerät, ${ }^{58}$ haben wir es im politischen Denken doch eher mit einer Entgrenzung zu tun. ${ }^{59}$ Die Ursachen hierfür sind vielfältig: Sie liegen zum einen in der imperialen Ideologie begründet, die den gesamten orbis terrarum in den Blick nimmt, zum andern aber auch in den konkreten Raumvorstellungen, die vor allem literarisch vermittelt und nur in Ansätzen kartographisch gestützt sind. ${ }^{60}$ Dies erschwert die dezidierte Auseinandersetzung mit Grenzverläufen, gerade in Regionen, in denen keine natürlichen Grenzen bestehen. ${ }^{61}$ Bedeutsam ist zudem, dass in den Randgebieten auch in der Kaiserzeit vielfach noch mit Formen indirekter Herrschaft wie Klientelkönigtümern gearbeitet wird. Auch das hemmt die Herausbildung einer Vorstellung von einer homogenen Grenze und ist einer Reflexion über die Ausmaße des Reiches nicht förderlich. ${ }^{62}$

Überlegungen zum Verzicht auf die Fortsetzung der Expansion, wie sie in der Kaiserzeit im ersten und zweiten Jahrhundert immer wieder begegnen, sind nicht programmatischer Natur, sondern situativen Charakters, werden etwa in Anbetracht tatsächlich erfahrener oder drohender Niederlagen formuliert und setzen auch nicht beim

\footnotetext{
${ }^{5}$ Dazu u. a. T.S. Burns, Rome and the Barbarians, 100 B. C. - A. D. 400, Baltimore/MD - London 2003, bes. 1-41; B. Isaac, The Barbarian in Greek and Latin Literature, in: ders., Empire and Ideology in the Graeco-Roman World. Selected Papers, Cambridge 2017, 197-220, hier 213-218.

${ }^{56}$ Einen kompakten Überblick über die verschiedenen Forschungsansätze und deren wichtigste Vertreter gibt A. Erskine, Roman Imperialism, Edinburgh 2010, 36-42. 47-49.

${ }^{57}$ Zur Sicherheit als zentralem Kriterium römischer Außenpolitik und entsprechenden Diskursen in Republik und Prinzipat Mattern, Rome and the Enemy, 215; speziell mit Blick auf die Kaiserzeit A. Kneppe, Metus temporum. Zur Bedeutung von Angst in Politik und Gesellschaft der römischen Kaiserzeit des 1. und 2. Jhdts. n. Chr., Stuttgart 1994, 217-281.

${ }^{58} \mathrm{Zu}$ dem Komplex mit Blick auf die augusteische Politik K.-W. Welwei, Probleme römischer Grenzsicherung am Beispiel der Germanenpolitik des Augustus, in: W. Schlüter/R. Wiegels (Hg.), Rom, Germanien und die Ausgrabungen von Kalkriese, Osnabrück 1999, 675-688, bes. 676.

${ }^{59} \mathrm{Zu}$ dieser Gemengelage C.R. Whittaker, Frontiers of the Roman Empire. A Social and Economic History, Baltimore/MD - London 1994, 68f.; A. Mehl, Imperium sine fine dedi - Die augusteische Vorstellung von der Grenzenlosigkeit des Römischen Reiches, in: E. Olshausen/H. Sonnabend (Hg.), Stuttgarter Kolloquium zur Historischen Geographie des Altertums. Bd. 4, Amsterdam 1994, 431-464, bes. 431-435. 463f.; E. Frézouls, La formation et l'évolution du concept de frontière à Rome, in: ibid., 465-486, bes. 479f.; G. Woolf, Inventing Empire in Ancient Rome, in: S. Alcock u. a. (Hg.), Empire. Perspectives from Archeology and History, Cambridge 2001, 311-322, hier 317-320; P. Edwell, Definitions of Roman Imperialism, in: D. Hoyos (Hg.), A Companion to Roman Imperialism, Leiden Boston 2013, 39-52, hier 45-51.

${ }^{60}$ Hier ist besonders an die sog. Karte des Agrippa zu denken, deren Charakter aber nach wie vor umstritten ist; dazu C. Nicolet, Space, Geography, and Politics in the Early Empire, Ann Arbor/Mich. 1991, 95-122; K. Brodersen, Terra cognita. Studien zur römischen Raumerfassung, Hildesheim 1995, 268-287; C. Hänger, Die Karte des Agrippa, in: M. Rathmann (Hg.), Wahrnehmung und Erfassung geographischer Räume in der Antike, Mainz 2007, 135-142, bes. 142.

${ }^{61}$ Vgl. Millar, Emperors, Frontiers and Foreign Relations, 31 B. C. to A. D. 378, 17; Whittaker, Frontiers of the Roman Empire, 68f.; Mattern, Rome and the Enemy, bes. 24f. 80.

${ }^{62} \mathrm{Zu}$ diesem Phänomen schon E. Kornemann, Die unsichtbaren Grenzen des römischen Kaiserreiches, in: ders., Gestalten und Reiche. Essays zur Alten Geschichte, Leipzig 1943, 323-338, bes. 323-326.
} 
Größenproblem an. ${ }^{63}$ Das gilt selbst für die Regentschaft Hadrians, die von der Forschung in dem Zusammenhang gern als Zäsur begriffen wird. ${ }^{64}$ Eine programmatische Neuorientierung der Politik, die der Einsicht in eine ,Überdehnung ' des Reiches Rechnung trüge, ist auch bei ihm nicht nachweisbar. ${ }^{65}$

Der Befund im zeitgenössischen politischen Denken ist ambivalent. Im Fokus steht gewöhnlich die Person des Kaisers; in Anbetracht des Ausmaßes seiner Macht, die nicht zuletzt auf der Größe des Reiches basiert, wird allem voran an dessen Bereitschaft, Maß zu halten, appelliert. Das gilt für griechische und lateinische Autoren gleichermaßen. ${ }^{66}$ Daneben sind aber auch Unterschiede zwischen den Schriftstellern auszumachen: Die lateinischen Autoren stehen mehrheitlich den senatorischen Kreisen nahe und erwarten von den principes militärisches Engagement und damit die Ausrichtung an traditionellen römischen Werten. Dies trifft auf Tacitus ebenso wie auf Plinius und Fronto zu, selbst auf Juvenal. Die Kritik an der hadrianischen Politik etwa setzt an der Stelle an, indem sie den ungenügenden Einsatz der Legionen und damit einhergehenden Disziplinverlust unter den Soldaten moniert. ${ }^{67}$ Letztlich geht es in dem Zusammenhang um Partizipationschancen für Senatoren und deren Relation zum princeps, nicht um gezielte Reflexionen zur Reichspolitik.

Bei griechischen Autoren stoßen wir demgegenüber auch auf Überlegungen, die explizit darauf zielen, auf weitere Expansion zu verzichten. Gleichwohl geht dies zumeist weder mit einer Absage an das imperiale Programm noch mit einer kritischen Auseinandersetzung mit der Größe des Reiches einher - ungeachtet der Tatsache, dass sich in jenen Texten speziell im Kontext der zweiten Sophistik zum Teil explizit romskeptische Aussagen finden. ${ }^{68}$ Gängig ist bei unserer Thematik vielmehr die Vorstellung, die römische Herrschaft umspanne bereits die gesamte Oikumene, so dass eine weitere Ausdehnung nur in unwirtliche Gegenden denkbar sei und sich damit verbie-

\footnotetext{
${ }^{63} \mathrm{Zu}$ dem Komplex D. Potter, The Limits of Power, in: D. Hoyos (Hg.), A Companion to Roman Imperialism, Leiden - Boston 2013, 319-332, hier 325-331.

${ }^{64} \mathrm{Zu}$ Umständen und Hintergründen K. Christ, Geschichte der römischen Kaiserzeit. Von Augustus bis zu Konstantin, München 1988, $316 f$.

${ }^{65}$ Der Widerstand gegen seine Politik, der sich insbesondere gegen die Aufgabe jener Provinzen richtet, die Trajan neu eingerichtet hatte, geht vor allem von Vertrauten und Heerführern Trajans aus, die von Hadrians Entscheidung massiv betroffen sind. Auf einen Grundsatzkonflikt in der Frage der Expansion ist daraus nicht zu schließen. Auch der Verfasser der Vita Hadriani in der Historia Augusta gibt keine Hinweise darauf; zu dessen Darlegung G. Kerler, Die Außenpolitik in der Historia Augusta, Bonn 1970, 25-37; M.T. Schmitt, Die römische Außenpolitik des 2. Jahrhunderts n. Chr., Stuttgart 1997, 63f.; G. Seelentag, Trajan, Hadrian und Antoninus Pius. Deutungsmuster und Perspektiven, in: A. Winterling (Hg.), Zwischen Strukturgeschichte und Biographie. Probleme und Perspektiven einer neuen Römischen Kaisergeschichte, München 2011, 295-315, hier 308f.; zu diesbezüglichen Kontroversen in der älteren Forschung siehe A.R. Birley, Roman Frontiers and Roman Frontier Policy. Some Reflections on Roman Imperialism, in: Transactions of the Architectural and Archaeological Society of Durham and Northumberland 3 (1974) 13-25, hier 15-19.

${ }^{66}$ Überdies trifft es auf verschiedene literarische Gattungen wie philosophische Werke, historiographische Schriften, aber auch panegyrische Texte in gleicher Weise zu. Es korrespondiert mit der Repräsentation des Kaisers, in der dieser Aspekt - insbesondere mit Blick auf die Erwartungen der Angehörigen des Senatorenstandes - ebenfalls stark herausgestrichen wird; dazu am Beispiel Trajans G. Seelentag, Taten und Tugenden Trajans. Herrschaftsdarstellung im Prinzipat, Stuttgart 2004, 21-23.

${ }^{67}$ Dazu mit Belegen aus Tacitus, Plinius und Fronto Schmitt, Die römische Außenpolitik des 2. Jahrhunderts n. Chr., 41-49.

${ }^{68}$ Sie stehen im Zusammenhang mit dem Selbstverständnis der Oberschichten in den Städten des griechischen Ostens, die den Verlust an politischer Handlungsfreiheit beklagen und sich ihrer paideia rühmen, durch die sie sich von den westlichen Eliten positiv abzugrenzen suchen; zu dem Komplex u. a. mit Blick auf Dion Chrysostomos T. Whitmarsh, Reading Power in Roman Greece. The Paideia of Dio Chrysostom, in: Y.L. Too/N. Livingstone (Hg.), Pedagogy and Power. Rhetoric of Classical Learning, Cambridge 1998, 192-213, hier 210-213.
} 
te. Auf Betrachtungen dieser Art stoßen wir beispielsweise in der ,Romrede ${ }^{6}$ des Aelius Aristides oder den Königsreden des Dion Chrysostomos, aber auch im Prooemium zu Appians ,Römischer Geschichte“ oder bei Cassius Dio. ${ }^{69}$ Teils begegnet hier der Gedanke, dass eine Fortsetzung der Expansion keinen Nutzen bringe, was in der Forschung z. T. dergestalt verstanden worden ist, dass nun ökonomische Maßstäbe in das politische Denken Einzug hielten und damit neue Motive für eine Selbstbeschränkung in der Reichspolitik generiert worden seien. ${ }^{70}$ Mir scheint das problematisch: Utilitaristische Argumente, die wir im politischen Denken seit dem fünften vorchristlichen Jahrhundert kennen, operieren zumeist nicht mit ökonomischen Kriterien. ${ }^{71}$ Kaiserzeitliche griechische Autoren verwenden den Begriff des $\sigma \nu \mu \varphi \varepsilon ́ \rho o v$ durchaus in mannigfaltiger Bedeutung, auch im Hinblick auf das Imperium Romanum $;{ }^{72}$ im vorliegenden Fall ist er eindeutig auf die Politik des Kaisers und die darauf bezogenen Erwartungen der Bevölkerung gerichtet: Nützlich ist demnach, was dem euergetischen Wirken des Kaisers förderlich scheint. ${ }^{73}$ Andere Schriftsteller, die den paxGedanken nicht in der Tradition der augusteischen Ideologie mit der Reichspolitik verknüpfen, sondern der Kriegführung generell eine Absage erteilen - etwa die Stoiker Musonius und Epiktet - praktizieren dies nicht auf der Basis einer Kritik am Imperium, sondern im Kontext einer Abwendung von politischen Fragen generell. ${ }^{74}$

In der Spätantike ist das Problem der ,Überdehnung' des Reiches aus moderner Sicht am virulentesten. Gerade in dieser Zeit aber wird es von den Zeitgenossen kaum reflektiert. Das politische Denken ist geprägt durch unterschiedliche Varianten der Romidee. ${ }^{75}$ Im Zuge der zunehmenden Idealisierung wie auch Transzendierung des Reiches verlieren realpolitisch orientierte quantifizierende Betrachtungen wie eine kritische Auseinandersetzung mit den Ausmaßen des Herrschaftsgebietes an Bedeutung. Der Umstand, dass Dekadenzvorstellungen nun in großer Zahl kursieren, ändert daran nichts. ${ }^{76}$ Diese Vorstellungen sind anders gelagert, operieren entweder mit dem Motiv eines ,natürlichen Alterungsprozesses` oder mit moralischen Kategorien, die herkömmlicher Provenienz wie auch christlich inspiriert sein können. ${ }^{77}$ Maßnahmen zur Schaffung kleinerer Einheiten, die administrativ und militärisch effektiver zu handhaben sind, namentlich die Reichsteilungen, die seit der Tetrarchie immer wieder praktiziert werden, werden im politischen Diskurs nicht als konstruktiver Rückbau verstanden, sondern als Abkehr von der traditionellen Reichsidee und damit als weite-

\footnotetext{
${ }^{69}$ Siehe z. B. Aristid. or. 26,10f.; Cassius Dio 52,14. 18. 37; 56,33,5f.; zu weiteren Belegen siehe F. Unruh, Das Bild des Imperium Romanum im Spiegel der Literatur an der Wende vom 2. zum 3. Jh. n. Chr., Bonn 1991, 15-26; Cornell, The End of Roman Imperial Expansion, 146f.

${ }^{70}$ So bei H. Gesche, Rom. Welteroberer und Weltorganisator, München 1981, 172.

${ }^{71}$ Im klassischen Athen begegnen sie im Bereich der Außenpolitik v. a. unter Machtgesichtspunkten; siehe beispielsweise aus attischen Demegorien Demosth. 1,1; 3,36; 4,51; 14,3; [Demosth.] 10,1. 17; dazu K. Piepenbrink, Politische Ordnungskonzeptionen in der attischen Demokratie des vierten Jahrhunderts v. Chr. Eine vergleichende Untersuchung zum philosophischen und rhetorischen Diskurs, Stuttgart 2001, 110.

${ }^{72}$ Dazu M. Hose, Erneuerung der Vergangenheit. Die Historiker im Imperium Romanum von Florus bis Cassius Dio, Stuttgart - Leipzig 1994, 386f. mit Blick auf Cassius Dio.

${ }^{73}$ Siehe bes. Dion Chrys. 1,27; 3,36; 4,44; zu dem Komplex Schmitt, Die römische Außenpolitik des 2. Jahrhunderts n. Chr., 22f.

${ }^{74}$ Vgl. H. Sidebottom, Philosophers' Attitudes to Warfare under the Principate, in: J. Rich/G. Shipley (Hg.), War and Society in the Roman World, London - New York 1993, 241-264, hier 245-250.

${ }^{75}$ Einschlägig ist hierzu noch immer M. Fuhrmann, Die Romidee der Spätantike, in: HZ 207 (1968) 529-561.

${ }^{76}$ Einen Überblick hierzu gibt Demandt, Der Fall Roms, bes. 67-70.

${ }_{77}$ Siehe etwa A. Demandt, Das Ende des Altertums in metaphorischer Deutung, in: Gymnasium 77 (1980) 178-204, hier 179f. (besonders zum Roma-senescens-Motiv; diesbezüglich speziell zur Spätantike Demandt, Der Fall Roms, 44-70; mit zahlreichen Quellen- und Literaturangaben).
} 
res Verfallsphänomen. ${ }^{78}$ Die Hauptverantwortung für militärische Niederlagen wie auch innere Krisen werden - wie schon im Prinzipat - beim jeweiligen Kaiser und dessen Helferstab gesehen und damit personalisiert. ${ }^{79}$ Nicht selten wird dabei auf die Korruption verwiesen, die aber nicht als Problem von administrativer Ausgestaltung und Kontrolle eines allzu großen Reiches perzipiert, ${ }^{80}$ sondern unter Rekurs auf das Paradigma der zunehmenden moralischen Depravation gedeutet wird. ${ }^{81}$

\section{Zusammenfassung}

Die Problematisierung von Größe im Hinblick auf Herrschaften ist in der Antike von geringerer Bedeutung als in der Rezeptions- und Wirkungsgeschichte, aber auch als in der modernen Forschung. Eine Parallele zwischen antiker Perzeption und späterem Denken begegnet gleichwohl in der Einschätzung, dass die partizipatorische Demokratie an unmittelbare Kommunikation gebunden ist, die man bis in jüngste Zeit noch gewöhnlich mit Kleinräumigkeit assoziiert hat.

Hinsichtlich der vermeintlichen ,Überdehnung‘ des Römischen Reiches sieht es anders aus: Der Gedanke ist in der Rezeptionsgeschichte verbreitet und findet sich aktuell besonders in populärwissenschaftlichen Arbeiten. In der Antike ist er demgegenüber nicht prominent. Das hat $u$. a. damit zu tun, dass hier - ungeachtet eines ausgeprägten Sendungsbewusstseins - nicht mit einer imperialen Konzeption gearbeitet wird, die pragmatisch ausgerichtet ist. Neuzeitliche Autoren, welche die antiken Verhältnisse reflektieren, gehen nicht von den antiken Handlungsbedingungen aus, sondern von jenen der eigenen Zeit. ${ }^{82}$ Sie sind zum einen durch das britische Empire und dessen Probleme, zum anderen durch Prinzipien moderner Staatlichkeit geprägt. Hiervon weicht das antike Imperium Romanum in vieler Hinsicht ab. ${ }^{83}$ Eine für unsere Thematik zentrale Differenz ist darin auszumachen, dass das Römische Reich - selbst in der Spätantike noch - einen vergleichsweise geringen Grad an Zentralisierung aufweist. Die Provinzen verfügen über ein hohes Maß an Eigenständigkeit, hinzu kommen die Selbstverwaltungsrechte der provinzialen Städte. Dies gilt - wenn auch mit

\footnotetext{
${ }^{78}$ Hierzu einschlägig A. Pabst, Divisio regni. Der Zerfall des Imperium Romanum aus der Sicht der Zeitgenossen, Bonn 1986, bes. 218-221; zu den praktischen Problemen, die nicht zuletzt darauf beruhen, dass am Gedanken der Reichseinheit stets festgehalten wird, B. Bleckmann, Bemerkungen zum Scheitern des Mehrherrschaftssystems: Reichsteilung und Territorialansprüche, in: A. Demandt u. a. (Hg.), Diokletian und die Tetrarchie. Aspekte einer Zeitenwende, Berlin - New York 2004, 74-94, bes. 91f.

${ }^{79}$ Personalisierte Ansätze finden sich teils auch in der modernen Forschung, hier aber weniger stark ethisiert und typisiert als mit Blick auf die Qualitäten einzelner Regenten besonders als Heerführer; so z. B. A. Ferrill, The Fall of the Roman Empire. The Military Explanation, London 1986, bes. 166; skeptisch hinsichtlich der Entscheidungsspielräume Einzelner M. Meier, Der Völkerwanderung ins Auge sehen. Individuelle Handlungsspielräume im 5. Jh., Heidelberg 2016, bes. 63.

${ }^{80} \mathrm{Zu}$ dem Phänomen R. MacMullen, Corruption and the Decline of Rome, New Haven - London 1988, bes. 148-167.

${ }^{81}$ Dazu mit Quellenbelegen seit der hohen Kaiserzeit K.D. Bracher, Verfall und Fortschritt im Denken der römischen Kaiserzeit. Studien zum Zeitgefühl und Geschichtsbewußtsein des Jahrhunderts nach Augustus, Wien - Köln - Graz 1987, bes. 191.

${ }^{82}$ Das gilt für Autoren der Aufklärung wie auch für solche des 19. Jahrhunderts gleichermaßen; dazu mit Quellenbelegen und weiteren Literaturhinweisen E.M. Hausteiner, Greater than Rom. Neubestimmungen britischer Imperialität 1870 - 1914, Frankfurt/M. u. a. 2015, 131-163; grundsätzlich auch S. Mills, Roman Imperialism. Critics and Aspirants, in: D. Hoyos (Hg.), A Companion to Roman Imperialism, Leiden - Boston 2013, 333-345.

${ }^{83}$ Eine Zusammenstellung wesentlicher Aspekte bietet D.J. Mattingly, Imperialism, Power, and Identity. Experiencing the Roman Empire, Princeton/N.J. 2013, 3-42.
} 
gewissen Unterschieden - für die Republik wie die Kaiserzeit gleichermaßen. ${ }^{84}$ Die Größe des Gesamtterritoriums gereicht hier in geringerem Ausmaß zum Problem als in Imperien mit einer stärker ausgeprägten zentralistischen Fokussierung. Entscheidend für Rom ist vielmehr die Integration der Herrschaftsträger und deren Ausrichtung auf römische Werte. ${ }^{85}$

Wenn übermäßige Größe von Herrschaftsgebieten im antiken politischen Denken kritisch gewürdigt wird, so geschieht das meist unter moralischen Gesichtspunkten mit Blick auf einzelne politisch Verantwortliche. ${ }^{86}$ Dabei werden nicht der Handlungsrahmen und dessen institutionelle Ausgestaltung ins Visier genommen, sondern das persönliche Verhalten maßgeblicher Akteure innerhalb dieses Rahmens. Ausnahmen hierzu finden wir in der klassischen Poliswelt, in der politische Gemeinwesen in einem für die Antike ungewöhnlichen Maße als ,gestaltbar' begriffen werden.

\footnotetext{
${ }^{84}$ Mit Blick auf die Republik R. Schulz, Herrschaft und Regierung. Roms Regiment in den Provinzen in der Zeit der Republik, Paderborn 1997, bes. 17; zur Kaiserzeit W. Eck, Die staatliche Administration des römischen Reiches in der hohen Kaiserzeit. Ihre strukturellen Komponenten, in: ders., Die Verwaltung des Römischen Reiches in der hohen Kaiserzeit. Ausgewählte und erweiterte Beiträge. Bd. 1, Basel - Berlin 1995, 1-28, bes. 1-6 (zuerst 1989); F.M. Ausbüttel, Die Verwaltung des Römischen Kaiserreiches. Von der Herrschaft des Augustus bis zum Niedergang des Weströmischen Reiches, Darmstadt 1998, 24-46.

${ }^{85}$ Das korreliert mit der jüngst von René Pfeilschifter formulierten These, dass die Expansion in Rom jenseits des Sittenverfalls-Diskurses - nicht grundsätzlich diskutiert worden sei; siehe Pfeilschifter, Eroberung und Debatte, bes. 152. 155.

${ }^{86}$ Diese müssen nicht als ,Individuen' konzipiert sein, sondern können - wie etwa in der späten römischen Republik - auch als Exponenten von Gruppen gezeichnet werden. Hinzu kommt eine ausgeprägte ,Typisierung'.
} 